\title{
A hybrid method for heartbeat classification via convolutional neural networks, multilayer perceptrons and focal loss
}

\author{
Tao Wang ${ }^{\text {Corresp., } 1}{ }^{,}$Changhua Lu $^{1}$, Mei Yang ${ }^{2}$, Feng Hong $^{1}$, Chun Liu ${ }^{\text {Corresp. } 3}$ \\ ${ }^{1}$ School of Computer and Information, Hefei University of Technology, Hefei, Anhui, China \\ 2 Beijing Huaru Technology Co., Ltd. Hefei Branch, Hefei, Anhui, China \\ 3 School of Electrical Engineering and Automation, Hefei University of Technology, Hefei, Anhui, China \\ Corresponding Authors: Tao Wang, Chun Liu \\ Email address: wtustc@mail.ustc.edu.cn, dqlch03@hfut.edu.cn
}

Background: Heart arrhythmia, as one of the most important cardiovascular diseases (CVDs), has gained wide attention in the past two decades. The paper proposes a hybrid method for heartbeat classification via convolutional neural networks, multilayer perceptrons and focal loss. Methods: In the method, a convolution neural network is used to extract the morphological features. The reason behind this is that the morphological characteristics of patients have inter-patient variations, which makes it difficult to accurately describe using traditional hand-craft ways. Then the extracted morphological features are combined with the RR intervals features and input into the multilayer perceptron for heartbeat classification. The RR intervals features contain the dynamic information of the heartbeat. Furthermore, considering that the heartbeat classes are imbalanced and would lead to the poor performance of minority classes, a focal loss is introduced to resolve the problem in the paper. Results: Tested using the MIT-BIH arrhythmia database, our method achieves an overall positive predictive value of $64.68 \%$, sensitivity of $68.55 \%$, f1-score of $66.09 \%$, and accuracy of $96.27 \%$. Compared with existing works, our method significantly improves the performance of heartbeat classification. Conclusions: Our method is simple yet effective, which is potentially used for personal automatic heartbeat classification in remote medical monitoring. The source code is provided on

https://github.com//ackAndCole/Deep-Neural-Network-For-Heartbeat-Classification . 
1 A hybrid method for heartbeat classification

2 via convolutional neural networks, multilayer

3 perceptrons and focal loss

4

5 Tao Wang ${ }^{1}$, Changhua $\mathrm{Lu}^{1}$, Mei Yang ${ }^{2}$, Feng Hong ${ }^{1}$ and Chun $\mathrm{Liu}^{3}$

6

$7{ }^{1}$ School of Computer and Information, Hefei University of Technology, Hefei, Anhui, China

$8 \quad 2$ Beijing Huaru Technology Co., Ltd. Hefei Branch, Hefei, Anhui, China

$9{ }^{3}$ School of Electrical Engineering and Automation, Hefei University of Technology, Hefei, Anhui,

10 China

11

12 Corresponding Author:

13 Chun Liu $^{3}$ and Tao Wang ${ }^{1}$

14 Fuicui Lake Campus of HeFei University of Technology, Hefei, Anhui, China

15 Email address: dqlch03@hfut.edu.cn (C. Liu) and wtustc@mail.ustc.edu.cn (T. Wang) 


\section{Abstract}

19 Background: Heart arrhythmia, as one of the most important cardiovascular diseases (CVDs), has gained wide attention in the past two decades. The paper proposes a hybrid method for heartbeat classification via convolutional neural networks, multilayer perceptrons and focal loss. Methods: In the method, a convolution neural network is used to extract the morphological features. The reason behind this is that the morphological characteristics of patients have interpatient variations, which makes it difficult to accurately describe using traditional hand-craft ways. Then the extracted morphological features are combined with the RR intervals features and input into the multilayer perceptron for heartbeat classification. The RR intervals features contain the dynamic information of the heartbeat. Furthermore, considering that the heartbeat classes are imbalanced and would lead to the poor performance of minority classes, a focal loss is introduced to resolve the problem in the paper.

Results: Tested using the MIT-BIH arrhythmia database, our method achieves an overall positive predictive value of $64.68 \%$, sensitivity of $68.55 \%$, f1-score of $66.09 \%$, and accuracy of $96.27 \%$. Compared with existing works, our method significantly improves the performance of heartbeat classification.

34 Conclusions: Our method is simple yet effective, which is potentially used for personal automatic heartbeat classification in remote medical monitoring. The source code is provided on https://github.com/JackAndCole/Deep-Neural-Network-For-Heartbeat-Classification.

\section{Introduction}

Heart arrhythmia, one of the most important cardiovascular disease (CVD), refers to the irregular beating of the patient's heart. Most arrhythmias are asymptomatic and not severe, but some could cause heart disease symptoms such as passing out, lightheadedness, chest pain, shortness of breath, and even stroke and cardiac arrest such as ventricular fibrillation, ventricular escape and atrial fibrillation, which are extremely dangerous and need immediate treatment. According to statistics from the World Health Organization (WHO), the number of CVD deaths in 2015 is close to 17.7 million, accounting for about $31 \%$ of the total deaths (Shen et al. 2019).

Electrocardiogram (ECG), a device that records the electrical activity of the heart, is widely used to diagnose cardiac arrhythmias in clinical (Mondéjar-Guerra et al. 2019). An ECG signal consists of a series of periodically repeating heartbeats. Each heartbeat usually contains a QRS complex, a $T$ wave, and a $P$ wave, in a few cases there is a $U$ wave (Vulaj et al. 2017). The most significant characteristic of an ECG signal is the QRS complex. By analyzing this complex, arrhythmia can be detected. However, the occurrence of arrhythmia is intermittent, especially in the early stages, 
51 which makes it difficult to perform effective detection in a short time (Mondéjar-Guerra et al. 52 2019).

53 To solve this problem, a Holter monitor is often used to collect long-term heart electrical activity 54 recordings (Sannino \& De Pietro 2018). In general, an ECG recording lasts several minutes or 55 even hours. Investigating a variety of abnormal arrhythmias beat-by-beat from long-term ECG 56 recordings is very exhausting, even for trained cardiologists. Therefore, there is an urgent need for 57 a computer-aided method to automatically detect abnormal heartbeats from long-term ECG data. 58 Over the past two decades, a lot of research works (de Albuquerque et al. 2018; De Chazal et al. 59 2004; Mondéjar-Guerra et al. 2019) have been spent on classifying heartbeats automatically. Most 60 of these methods are based on morphological characteristics of heartbeats and traditional signal 61 processing techniques. However, the ECG waveform and its morphological characteristics (e.g. 62 the shape of the QRS waves and $\mathrm{P}$ wave) of different patients are significantly different, and for 63 the same patient, there are also differences in different circumstances (Mondéjar-Guerra et al. 64 2019), so the fixed features used in these methods are not sufficient to accurately distinguish 65 arrhythmias for all patients. In recent years, some deep neural networks have been proposed, such 66 as convolutional neural networks $(\mathrm{CNN})$, which can automatically extract morphological features 67 and adapt to variations between patients.

68 Nevertheless, there is another challenge when processing medical data. Due to the limited number 69 of rare classes, the number of one class may greatly exceed that of other classes, that is, the 70 distribution of classes is imbalanced. However, most algorithms try to minimize the overall 71 classification loss during the training process, which implies that these classes are equally 72 important and the same misclassification cost is allocated to all types of errors. As a result, the 73 classifier will tend to correctly classify and favor more frequent classes.

74 The paper presents a hybrid method for heartbeat classification via CNN, multilayer perceptrons 75 (MLP) and focal loss. An overall structure of the method is displayed in Fig. 1. The morphological 76 features are extracted by one-dimensional (1D) $\mathrm{CNN}$ and combined with the RR intervals features 77 as the input of MLP. The RR intervals features contain the dynamic information of the heartbeat, 78 which could help better capture the pattern of the ECG waveform. Furthermore, considering that 79 the heartbeat classes are imbalanced and would lead to the poor performance of minority classes, 80 a focal loss is introduced to solve the problem. It shows superior performance in various 81 application environments (Howland et al. 2002; Lin et al. 2017; Zhou et al. 2002). By testing in 82 the well-known MIT-BIH arrhythmia database (Moody et al. 2001), our method achieves superior 83 classification performance than existing heartbeat classification methods. Note that the accuracy 84 of the ECG classification method has been standardized according to AAMI's recommendations 
85 (the Association for the Advancement of Medical Instrumentation). The proposed method obtains 86 an overall PPV of $64.68 \%$, SE of $68.55 \%$, F1 of $66.09 \%$, and accuracy of $96.27 \%$.

87 The paper is organized as follows: Section II presents the related works of heartbeat classification.

88 The proposed method and loss function are introduced in Section III and IV. The dataset and the 89 performance of our method against existing works are described in Section V. Section VI discusses 90 the conclusions.

\section{Related works}

The existing automatic heartbeat classification works can be divided into two paradigms: intrapatient paradigm and inter-patient paradigm (De Chazal et al. 2004; Sannino \& De Pietro 2018). In the intra-patient paradigm, the dataset is based on the heartbeat label split into training and test subsets, so an ECG recording will appear in two subsets (Sannino \& De Pietro 2018). According to de Chazal et al. (De Chazal et al. 2004), the results of this paradigm are biased, resulting in an accuracy of about $100 \%$ in the test phase, because the patient's characteristics are learned during the training phase (Sellami \& Hwang 2019). However, in actual scenarios, the trained model must be able to handle inter-patient variations during the training phase. In the inter-patient paradigm, the training set and test set are from different patients (Sannino \& De Pietro 2018), so the differences between patients will be considered during the training process. The classifier will show a better generalization capability. For instance, de Chazal et al. (De Chazal et al. 2004) propose a linear discriminant heartbeat classification method based on heartbeat morphological and dynamic features. Their method achieves a PPV of $38.5 \%$, SE of $75.9 \%$ in the SVEB class, and a PPV of $81.6 \%$, SE of $80.3 \%$ in the VEB class. Ye et al. (Ye et al. 2012) apply wavelet transform and independent component analysis (ICA) to extract morphological features from heartbeats, and combined with dynamic RR interval features develop an SVM method to classify heartbeat. A PPV of $52.3 \%$, SE of $60.8 \%$ in the SVEB class, and a PPV of $63.1 \%$, SE of $81.5 \%$ in the VEB class are obtained by their method. However, the classification accuracies of these methods are significantly lower than the intra-patient paradigm-based methods. This is due to variations of ECG characteristics between patients.

112 Recently, with the rapid development in deep learning, deep neural networks-based, especially

113 CNN-based, heartbeat classification methods have received a lot of attention. For example,

114 Yildırım et al. (Yildırım et al. 2018) develop a 1D-CNN for arrhythmia detection based on long-

115 term ECG signal. Their method achieves 91.33\% overall accuracy in 17 cardiac arrhythmias.

116 Similarly, Sellami et al. (Sellami \& Hwang 2019) develop a CNN with a batch-weighted loss

117 function for heartbeat classification. Hannun et al. (Hannun et al. 2019) present a deep neural 118 network with residual block to classify 12 rhythm classes. Romdhane et al. (Romdhane et al. 2020) 
119 based on CNN and focal loss propose an ECG heartbeat classification method. Although the 120 performance of heartbeat classification is improved, these works mainly focus on using CNN to 121 extract the heartbeat morphological features, while ignoring the influence of RR intervals on

122 heartbeat classification. Research shows that by integrating RR interval features, the performance

123 of heartbeat classification can be significantly improved (De Chazal et al. 2004; Mondéjar-Guerra

124 et al. 2019; Sannino \& De Pietro 2018). Romdhane et al. (Romdhane et al. 2020) try to use an

125 improved heartbeat segmentation method to make CNN capture RR interval information, but in

126 their work, CNN can only extract the previous RR interval information at most. This is due to the

127 incomplete division of the right interval. Different from existing works, we pre-extract the RR

128 interval information in advance, and then combine it with CNN-based morphological features as

129 the input of the classifier.

130 In addition to the above two classification paradigms, a hybrid paradigm has also been studied by

131 some scholars, namely patient-specific paradigm. In the patient-specific paradigm, a global model

132 is first built and then use part of patient data to tune the model to form a local model. de Chazal et

133 al. shows that this paradigm is superior to a pure inter-patient model. However, this paradigm

134 requires a professional doctor to label part of the ECG data, and an engineer to fine-tuning the

135 model in clinical. Meanwhile, the patient's ECG signal may change significantly over time, that is,

136 the current ECG signal may undergo large variations at some time in the future, and the use of a

137 previously fine-tuned local classifier may lead to larger misclassification. We focus on the

138 performance of our method in the inter-patient paradigm in the paper.

139 Methods

140 Fig. 1 shows the overall structure of the proposed method. The proposed method includes three

141 steps: ECG denoising, feature extraction, and classification. The feature extraction step contains

142 RR intervals features extraction and morphological features extraction via CNN architecture.

\section{ECG denoising}

144 The ECG signal is usually disturbed by various noises such as electromyography noise, power line

145 interference and baseline wandering (Chen et al. 2017), which makes useful features to be

146 difficultly extracted. In this step, most previous works typically perform a baseline wandering

147 removal and then high-frequency noise filtering (Mondéjar-Guerra et al. 2019). However,

148 excessive filtering will lead to the loss of some helpful information in the ECG signal. Since CNN

149 has better noise immunity (Huang et al. 2018), we only perform baseline wandering removal and

150 preserve as much information as possible from the raw ECG signal.

151 Two median filters are combined to remove the baseline wandering of the ECG signal in the paper.

152 First, the QRS complexes and P-waves are removed using a 200-ms width median filter, and then 
153 a 600-ms width median filter is further adopted to remove T-waves. The output is the baseline

154 wandering of the ECG signal, and the baseline-corrected ECG signal can be achieved by

155 subtracting it from the original signal. An effect of baseline wandering removal is shown in Fig.

1562.

157 After obtaining the baseline-corrected ECG signal, the ECG is further segmented into a series of

158 heartbeats based on the labeled R-peaks. In specific, for each heartbeat, we obtain 200 sampling

159 points of the ECG signal segment, 90 sampling points before and 110 sampling points after the

160 labeled $\mathrm{R}$ peak. The R-peak detection is not the focus of the paper and we directly use labeled R-

161 peaks in the dataset, as there are many high-precision ( $>99 \%)$ R-peak detection methods in the

162 literature (Adam 2012; Pan \& Tompkins 1985).

\section{RR Interval features extraction}

164 The time interval between two consecutive R-peaks is normally called the RR interval 165 (Ruangsuwana et al. 2010), which contains the dynamic information of the heartbeat. To capture 166 this information for heartbeat classification, four features are extracted from the RR interval, 167 namely previous RR interval, post RR interval, ratio RR and local RR interval. The previous RR 168 interval refers to the distance between the current R-peak position and the previous R-peak, the 169 post RR interval is the distance between the current R-peak position and the following one. The 170 ratio $R R$ represents the ratio of the previous $R R$ interval and the post $R R$ interval. These three 171 features reflect the instantaneous rhythm of a heartbeat. The average value of the $10 \mathrm{RR}$ intervals 172 before the current heartbeat is taken as the local RR interval, which represents the overall rhythm 173 in the past. Due to the inter-patient variations in the ECG signal, the RR interval of different 174 patients cannot be directly compared, in this paper we use the entire patient's ECG signal to 175 calculate the average RR interval, and subtract it from all RR characteristics (expect the ratio RR) 176 to eliminate this effect.

\section{Morphological features extraction via CNN architecture}

$178 \mathrm{CNN}$ is a powerful deep neural network inspired by visual neuroscience (Chu et al. 2019b). It has 179 been successfully used in speech recognition, natural language processing, image classification, 180 and biomedical signal (Palaz \& Collobert 2015; Pourbabaee et al. 2018; Yin et al. 2017). Given an 181 image, CNN can effectively learn high-level abstractions, which can then be input into the 182 classifier (e.g. fully connected neural network and support vector machine (SVM)) for 183 classification (Zhang et al. 2017). A CNN usually consists of convolutional layers, activation 184 functions, and pooling layers, and sometimes including batch normalization layers. 
185 Convolutional Layer: It is the most important component in CNN and performs convolution 186 operation on the input data (Liu \& Chen 2017). Let $f_{k}$ and $\mathrm{s}$ be the filter and the 1D ECG signal, 187 respectively. The output of the convolution is calculated as follows:

$$
C[i]=s(i) * f_{k}(i)=\sum_{m} s(m) f_{k}(i-m)
$$

189

where $m$ is the size of the filter and the filter $f_{k}$ is realized by sharing the weights of adjacent neurons.

Activation Function: The activation function is used to determine whether the neuron should be activated. The purpose is to enable neurons to achieve nonlinear classification. Rectifier Linear Unit (ReLU) is one of the most widely used activation function, which can be expressed as

$$
f(x)=\max (0, x)
$$

where $x$ is the output value of the neuron.

Pooling layer: The pooling layer, also known as the down-sampling layer, is an operation that decreases the computational intensity by reducing the output neuron dimension of the convolutional layer, and can handle some variations due to signal shift and distortion (Zhang et al. 2017). The most widely used pooling method is the max-pooling, which is to apply the maximum function over input $s$. Let $m$ be the filter size, and the output is:

Batch Normalization Layer: The batch normalization layer is a technology for standardizing network input, applied to either the activations of a prior layer or inputs directly, which can accelerate the training process, and provides some regularization, reducing generalization error. Let $B=\left\{x_{i}, i=1, \cdots, m\right\}$ be a mini-batch of the entire training set, the output of batch normalization is as follows:

$$
M(x)=\max \left\{s(x+k)|| k \mid \leq \frac{m-1}{2}\right\}
$$

$$
\hat{x}_{i}=\frac{x_{i}-\mu_{B}}{\sqrt{\sigma_{B}^{2}+\epsilon}}
$$

where $\sigma_{\mathrm{B}}$ and $\mu_{B}$ are the variance and the mean of training set $B$, respectively. $\epsilon$ is an arbitrarily small constant to ensure the denominator is not zero.

210 A CNN is developed and utilized for heartbeat morphological feature extraction in this paper. The $211 \mathrm{CNN}$ architecture is displayed in Fig. 1. It contains 3 convolutional blocks and 3 pooling layers.

212 Each convolutional block includes a convolution layer, a ReLU activation function and a batch 213 normalization layer. The kernel of the convolution is reduced as the network structure becomes 214 deeper. For instance, the first convolution kernel is 11, while the second is reduced to 5. A batch 215 normalization and ReLU activation are applied after each convolution operation, and a max- 
216 pooling is used to reduce the spatial dimension. Note that the parameters of the convolutional

217 network are usually set based on the author's experience. The detailed parameters of CNN

218 architecture are listed in Table 1. The output of the last pooling layer is the morphological features

219 extracted by CNN from the heartbeat. An illustration of the morphological features extracted from

220 the heartbeat is shown in Fig. 1 (c).

221 MLP classifier

222 The CNN-based morphological features and RR interval features are combined as the input of the

223 classifier in the paper. In general, any classifier (i.e. support vector machine (SVM) and random

224 forest $(\mathrm{RF})$ ) can be used for heartbeat classification. Here, we adopt a multilayer perceptron (MLP,

225 also known as fully connected neural networks in deep learning) as the classifier. The reason

226 behind this is that CNN and MLP can be combined for parameter training (we call it one-step

227 training). Compared with other methods, this usually achieves better performance. Specifically,

228 our MLP classifier contains an input layer, a hidden layer and an output layer. The input layer

229 consists of two parts of information: CNN-based morphological features and RR interval features.

230 The hidden layer has 64 neurons, and each neuron is connected to the input features. The output

231 layer neurons are 4 in the paper, each representing a kind of arrhythmia or normal heartbeat. The

232 details of our method are shown in Fig. 1 and Table 1.

233 Loss function

234 Before training a deep neural network, a loss function is first needed. The cross-entropy loss is the 235 most widely used in deep neural network classification (Chu et al. 2019a). However, this loss

236 function does not address the class imbalance problem. A focal loss function is introduced in the

237 paper to deal with this problem.

238 Cross-entropy loss

239 The cross-entropy is a measure in information theory (Robinson et al. 2001). It is based on entropy 240 and calculates the difference between two probability distributions. Closely related to KL 241 divergence that computers the relative entropy between two probability distributions, but the cross-

242 entropy calculates the total entropy between the distributions. The cross-entropy is usually taken 243 as the loss function in deep neural network classification (Chu et al. 2019a).

244 Let $t_{i}$ and $p_{i}$ be the ground truth and the estimated probability of each category, the cross-entropy 245 loss is computed by:

$$
C E=-\sum_{i}^{C} t_{i} \cdot \log \left(p_{i}\right)
$$


247

248

249

250

251

252

253

254

255

256

257

258

259

260

261

262

263

264

265

266

267

268

269

270

271

272

273

274

275

276

where $C$ refers to the category set of the heartbeat. In the cross-entropy loss, each category is treated equally, which causes the majority category to overwhelm the loss and the model tends to classify to the majority category in an imbalanced environment.

\section{Focal loss}

A characteristic of cross-entropy loss is that even easy-to-classify examples can cause significant losses, which will cause the loss of easy examples that constitute most of the dataset during the training process to negatively affect rare classes (Lin et al. 2017). The focal loss is designed to deal with this imbalanced problem by reshaping the cross-entropy loss function by reducing the attention to easy examples and focusing on difficult ones. A general formula for focal loss is expressed as:

$$
F L=-\sum_{i}^{C} t_{i} \cdot\left(1-p_{i}\right)^{\gamma} \log \left(p_{i}\right)
$$

where $\gamma$ acts as the modulating factor. As shown in Fig. 3, the higher the $\gamma$ value, the lesser the cost incurred by well-classified examples. In practice, the $\alpha$-balanced variant of the focal loss is usually used when one or more categories are highly imbalanced, which is defined as:

$$
F L^{\prime}=-\sum_{i}^{C} t_{i} \cdot \alpha_{i}\left(1-p_{i}\right)^{\gamma} \log \left(p_{i}\right)
$$

where $\alpha_{i}$ is the weighting factor of each category.

\section{Results}

\section{Data set}

The ECG dataset from the MIT-BIH arrhythmia database (Moody et al. 2001) is used to test our proposed method. This dataset contains 48 30-minute ambulatory two leads ECG signal records collected from 47 subjects. Each ECG signal is sampled at $360 \mathrm{~Hz}$ with an 11-bit resolution. The first lead is the modified-lead II (ML II), and the second lead depends on the record, one of V1, V2, V4 or V5. The heartbeat of these ECG signals is independently labeled by two or more doctors, and there are about 110,000 heartbeats.

According to the recommendation of the Association for the Advancement of Medical Instrumentation (AAMI), these heartbeats are further divided into five heartbeat classes. Table 2 shows the mapping of AAMI classes and MIT-BIH arrhythmia heartbeat types. Since Q is practically non-existent, we ignore it like others (Mar et al. 2011; Zhang et al. 2014). Meanwhile, four recordings with paced beats are removed in consistent with the AAMI recommended practice, namely 102, 104, 107 and 217. Since all records have ML II ECG signals and they are widely used 
277 in wireless body sensor network (WBSN) based ECG applications, this lead ECG signal is used

278 for heartbeat classification in the paper.

279 As we mentioned in related works, the paper focuses on the heartbeat classification under the inter-

280 patient paradigm. To facilitate comparison with existing works, we follow de Chazal et al. (De

281 Chazal et al. 2004) to split the dataset into two subsets. Each contains regular and complex

282 arrhythmia records and has roughly the same number of heartbeat types. Table 3 shows the details

283 of two subsets. The first (DS1) is used for training whereas the second (DS2) is used to test the

284 heartbeat classification performance (De Chazal et al. 2004). No patient appears in both subsets at

285 the same time.

\section{Model training and Performance metrics}

287 In the study, the general focal loss (non- $\alpha$-balanced focal loss) is used as the loss function, and the

288 modulating factor $\gamma$ is set to the default value $(\gamma=2)$. Since Adam can accelerate the model training,

289 we use it as the optimizer. The batch size of the model is set to 512 and the maximum epoch is 50.

290 The initial learning rate is 0.001 , and reduced by 0.1 times every 10 epochs. In addition, in order

291 to avoid overfitting, the 12 penalty is set to $1 \mathrm{e}-3$ based on trial and error. The model is implemented

292 using Keras and trained on the NVIDIA GeForce RTX 2080Ti graphical processing unit.

293 To evaluate the performance of our proposed method, three widely used metrics are adopted,

294 namely positive predictive value (PPV), sensitivity (SE), and accuracy (ACC), which are defined 295 as:

$$
P P V_{i}=\frac{T P_{i}}{T P_{i}+F P_{i}}
$$

$$
S E_{i}=\frac{T P_{i}}{T P_{i}+F N_{i}}
$$

where $T P_{i}$ (true positive) refers to the number of the $i$ th class is correctly classified, $F P_{i}$ (false positive) is equal to the number of heartbeats misclassified as the ith class, $T N_{i}$ (true negative) is the number of heartbeats that are not in the ith class and not classified into the ith class, and $F N_{i}$ (false negative) is equal to the number of heartbeats of the $i$ th class classified as other classes. $P P V_{i}$ indicates the proportion of positive correct classification, and $S E_{i}$ reflects the sensitivity of the classifier in the ith class. $A C C_{i}$ is the ratio of all correct classifications. 
305 Since the heartbeat classes are imbalanced, $f 1$-score $(F 1)$ is also selected as the performance 306 measure, defined as:

$$
F 1_{i}=\frac{2 \times P P V_{i} \times S E_{i}}{P P V_{i}+S E_{i}}
$$

308

309

310

311

312

313

314

315

316

317

318

319

320

321

322

323

324

325

326

327

328

329

330

331

332

333

334

335

336

$f 1$-score takes both the positive predictive value $P P V_{i}$ and sensitivity $S E_{i}$ into account, and is generally useful than $A C C_{i}$ in the imbalance class distribution (Chen 2009).

\section{Comparison with existing works}

Based on de Chazal et al. (De Chazal et al. 2004), the dataset is divided into DS1 and DS2 datasets. DS1 is used for training and DS2 is used to test our proposed method. For fair evaluation, we compare works (Chen et al. 2017; De Chazal et al. 2004; Garcia et al. 2017; Liu et al. 2019; Mar et al. 2011; Zhang et al. 2014) that adopt the same strategy. As SVEB and VEB are more important than other classes, we list the detailed information of these two classes in Table 4. The experimental results show that the proposed method has better recognition in the inter-patient paradigm, with F1s of SVEB and VEB of $74.29 \%$ and $92.40 \%$, respectively. In particular, the PPV of SVEB is $68.34 \%$, indicating that the proposed method has better SVEB recognition ability. The $93.72 \%$ SE of VEB is superior to most reported works. The evaluation results of all four-classes are listed in Table 5. The results related to PPV and SE are close to or surpass those obtained with existing works except for F. For category F, it is mainly composed of the fusion of ventricular beat and normal beat, which is very close to the normal heartbeat. Meanwhile, compared with other categories, $\mathrm{F}$ has the least number and the most serious imbalance. As a result, the performance of existing works is unstable in this category, usually a large number of $\mathrm{N}$ is predicted as $\mathrm{F}$ or $\mathrm{F}$ is predicted as N. In the paper, although the focus loss is introduced, due to the high imbalance of F, the proposed method cannot extract the discriminate features. Mar et al., although, obtains the best PPV in F, a large number of $\mathrm{N}$ is incorrectly classified as $\mathrm{F}$. We suggest that category $\mathrm{F}$ can be included in other categories in future research.

\section{Discussion}

\section{Focal loss vs. Cross-entropy loss}

Since the heartbeat has an imbalanced class distribution, the cross-entropy loss is replaced by the focal loss as the loss function of the model in the paper. The performance comparison of the two losses is listed in Table 6. Both losses have similar overall accuracy, but compared to cross-entropy loss, the overall PPV, SE and F1 of the focal loss are significantly improved. An overall PPV of $64.68 \%$, SE of $68.55 \%$, and F1 of $66.09 \%$ are achieved by the focal loss, while the cross-entropy loss obtains an overall PPV of $59.82 \%$, SE of $66.77 \%$, and F1 of $62.67 \%$. The corresponding 
337 metrics increased by $4.86 \%, 1.78 \%$, and $3.42 \%$, respectively. In addition, for each specific class,

338 the PPV, SE, and F1 of the focal loss also have achieved comparable or better performance than

339 the cross-entropy loss, especially in the F1.

340 The confusion matrix of the two losses is listed in Table 7. The focal loss achieves a total of 45,952

341 correct predictions, while the cross-entropy obtains 45,358 correct predictions. With the focal loss,

342 the total correct prediction has slightly increased, perhaps due to the suppression of easy-to-

343 classify samples by focal loss.

\section{Conclusions}

345 A hybrid method for heartbeat classification via CNN, MLP and focal loss is developed in the

346 paper. Among them, $\mathrm{CNN}$ is used to extract the morphological features of the heartbeat. Then the

347 morphological features are combined with the RR intervals features and input into the MLP to

348 perform heartbeat classification. Furthermore, in order to avoid the impact of heartbeat imbalance,

349 a focal loss function is introduced. Tested by using the MIT-BIH arrhythmia database, the

350 experimental results confirm that the method has good overall performance, with F1 of $66.09 \%$

351 and accuracy of $96.27 \%$. The superiority of the proposed method is due to multifactorial: (I)

352 Compared with traditional hand-craft features, $\mathrm{CNN}$ as an automatic extraction method can adapt

353 to small mutations in ECG signals to obtain powerful features; (II) Besides the CNN-based

354 morphological features, the pre-extracted RR interval features are also combined to build the

355 model, avoiding the loss of dynamic information due to heartbeat segmentation; (III) A focal loss

356 function is introduced to solve the class imbalance, preventing the model from biasing towards the

357 majority class; (IV) One-step training can improve the model to obtain better feature abstraction

358 capabilities. Due to the simple yet effective of the proposed inter-patient method, it has the

359 potential to be used for personal automatic heartbeat classification for surveillance in telemedicine.

360 The encouraging results have inspired continuous exploration. The future work will include (I)

361 testing the performance of the developed model with more ECG signals; (II) designing or

362 modifying CNN architecture to further improve the performance of our method; (III) trying to use

363 additional techniques such as wavelet transform to convert time-domain information to frequency-

364 domain information to reduce the difficulty of CNN feature extraction.

\section{References}

366 Adam GJBE. 2012. ECG Signal Processing, Classification and Interpretation.278.

367 Chen S, Hua W, Li Z, Li J, Gao XJBSP, and Control. 2017. Heartbeat classification using projected and dynamic features of ECG signal. 31:165-173.

Chen YJDoCS, Iowa State University. 2009. Learning classifiers from imbalanced, only positive and unlabeled data 370 sets. 
371 Chu J, Wang H, Lu WJJoMiM, and Biology. 2019a. A novel two-lead arrhythmia classification system based on CNN 372 and LSTM. 19:1950004.

373 Chu Y, Shen H, and Huang KJIA. 2019b. ECG authentication method based on parallel multi-scale one-dimensional 374 residual network with center and margin loss. 7:51598-51607.

375 de Albuquerque VHC, Nunes TM, Pereira DR, Luz EJdS, Menotti D, Papa JP, Tavares JMRJNC, and Applications. 2018. Robust automated cardiac arrhythmia detection in ECG beat signals. 29:679-693.

377

378

379

380

381

382

383

384

385

386

387

388

389

390

391

392

393

394

395

396

397

398

399

400

401

402

403

404

405

406

407

408
De Chazal P, O'Dwyer M, and Reilly RBJItobe. 2004. Automatic classification of heartbeats using ECG morphology and heartbeat interval features. 51:1196-1206.

Garcia G, Moreira G, Menotti D, and Luz E. 2017. Inter-Patient ECG Heartbeat Classification with Temporal VCG Optimized by PSO. Scientific Reports 7:10543. 10.1038/s41598-017-09837-3

Hannun AY, Rajpurkar P, Haghpanahi M, Tison GH, Bourn C, Turakhia MP, and Ng AY. 2019. Cardiologist-level arrhythmia detection and classification in ambulatory electrocardiograms using a deep neural network. Nature Medicine 25:65-69. 10.1038/s41591-018-0268-3

Howland DS, Liu J, She Y, Goad B, Maragakis NJ, Kim B, Erickson J, Kulik J, DeVito L, and Psaltis GJPotNAoS. 2002. Focal loss of the glutamate transporter EAAT2 in a transgenic rat model of SOD1 mutant-mediated amyotrophic lateral sclerosis (ALS). 99:1604-1609.

Huang J, Liu H, Dai J, and Cai W. 2018. Reconstruction for limited-data nonlinear tomographic absorption spectroscopy via deep learning. $\quad 218: 187.10 .1016 /$ j.jqsrt.2018.07.011

Lin T-Y, Goyal P, Girshick R, He K, and Dollár P. 2017. Focal loss for dense object detection. Proceedings of the IEEE international conference on computer vision. p 2980-2988.

Liu J, Song S, Sun G, and Fu Y. 2019. Classification of ECG Arrhythmia Using CNN, SVM and LDA. Cham: Springer International Publishing. p 191-201.

Liu Y, and Chen Y. 2017. Recognition of facial expression based on CNN-CBP features. 2017 29th Chinese Control And Decision Conference (CCDC): IEEE. p 2139-2145.

Mar T, Zaunseder S, Martínez JP, Llamedo M, and Poll RJItoBE. 2011. Optimization of ECG classification by means of feature selection. $58: 2168-2177$.

Mondéjar-Guerra V, Novo J, Rouco J, Penedo MG, Ortega MJBSP, and Control. 2019. Heartbeat classification fusing temporal and morphological information of ECGs via ensemble of classifiers. 47:41-48.

Moody GB, Mark RGJIEiM, and Magazine B. 2001. The impact of the MIT-BIH arrhythmia database. 20:45-50.

Palaz D, and Collobert R. 2015. Analysis of cnn-based speech recognition system using raw speech as input. Idiap.

Pan J, and Tompkins WJJItobe. 1985. A real-time QRS detection algorithm.230-236.

Pourbabaee B, Roshtkhari MJ, Khorasani KJIToS, Man, and Systems C. 2018. Deep convolutional neural networks and learning ECG features for screening paroxysmal atrial fibrillation patients. 48:2095-2104.

Robinson S, Cattaneo A, and El-Said MJESR. 2001. Updating and estimating a social accounting matrix using cross entropy methods. 13:47-64.

Romdhane TF, Alhichri H, Ouni R, and Atri M. 2020. Electrocardiogram heartbeat classification based on a deep convolutional neural network and focal loss. Computers in Biology and Medicine 123:103866. https://doi.org/10.1016/j.compbiomed.2020.103866

Peerj Comput. Sci. reviewing PDF | (CS-2020:08:52443:2:1:NEW 6 Nov 2020) 
409 Ruangsuwana R, Velikic G, and Bocko M. 2010. Methods to extract respiration information from ECG signals. 2010 IEEE International Conference on Acoustics, Speech and Signal Processing: IEEE. p 570-573.

411 Sannino G, and De Pietro GJFGCS. 2018. A deep learning approach for ECG-based heartbeat classification for $412 \quad$ arrhythmia detection. 86:446-455.

413 Sellami A, and Hwang HJESwA. 2019. A robust deep convolutional neural network with batch-weighted loss for 414 heartbeat classification. 122:75-84.

415 Shen R, Yu Y, Lan R, Yu R, Yuan Z, and Xia ZJEP. 2019. The cardiovascular toxicity induced by high doses of 416 gatifloxacin and ciprofloxacin in zebrafish. 254:112861.

417 Vulaj Z, Draganić A, Brajović M, and Orović I. 2017. A tool for ECG signal analysis using standard and optimized $418 \quad$ Hermite transform. 2017 6th Mediterranean Conference on Embedded Computing (MECO): IEEE. p 1-4.

419 Ye C, Kumar BV, and Coimbra MTJIToBE. 2012. Heartbeat classification using morphological and dynamic features 420 of ECG signals. 59:2930-2941.

Yıldırım Ö, Pławiak P, Tan R-S, and Acharya UR. 2018. Arrhythmia detection using deep convolutional neural network with long duration ECG signals. Computers in Biology and Medicine 102:411-420. https://doi.org/10.1016/j.compbiomed.2018.09.009

Yin W, Kann K, Yu M, and Schütze HJapa. 2017. Comparative study of cnn and rnn for natural language processing.

Zhang Q, Zhou D, and Zeng XJIA. 2017. HeartID: A multiresolution convolutional neural network for ECG-based biometric human identification in smart health applications. 5:11805-11816.

Zhang Z, Dong J, Luo X, Choi K-S, Wu XJCib, and medicine. 2014. Heartbeat classification using disease-specific feature selection. $46: 79-89$.

Zhou F-Q, Waterman-Storer CM, and Cohan CSJTJocb. 2002. Focal loss of actin bundles causes microtubule redistribution and growth cone turning. 157:839-849.

431 
Figure 1

A scheme of our proposed method.

a) Overview of our method. b) CNN block of our CNN architecture. c) An example of morphological features extracted by CNN, where the upper part heartbeat signal is the input of CNN, and the lower part is the features extracted by CNN, that is, the morphological features. These features will be flattened and combined with the RR interval features when used as the input of the MLP classifier.

a.

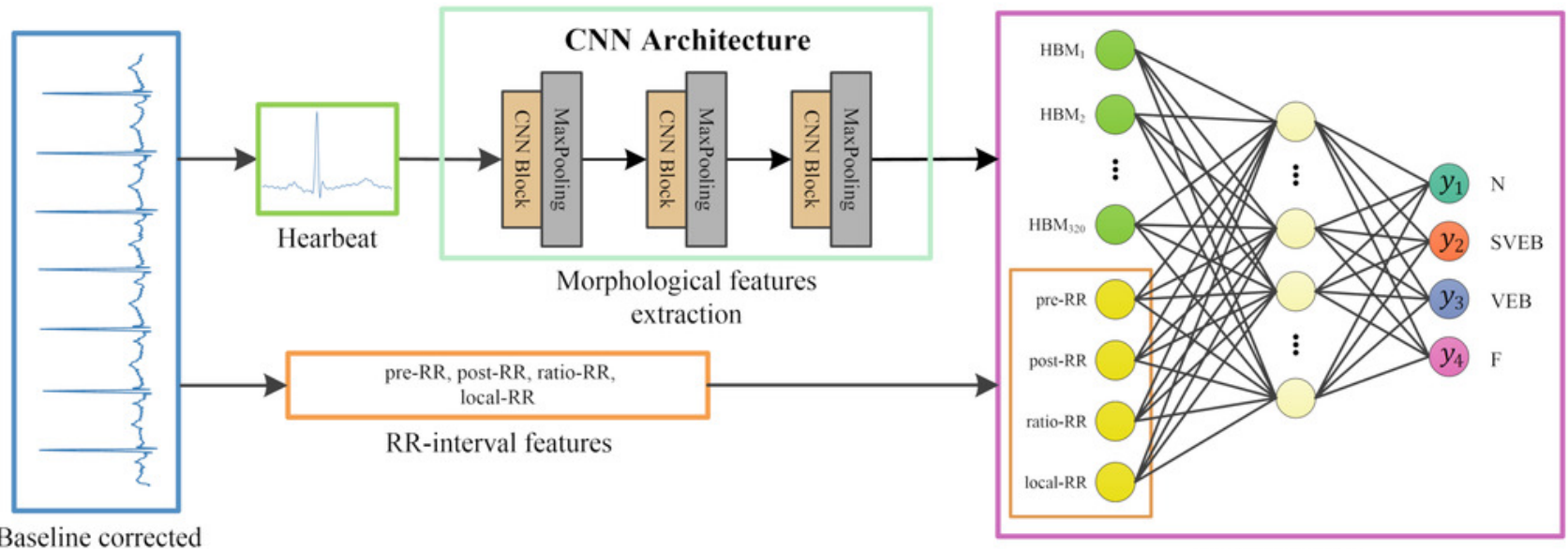

Baseline corrected

b.

ECG Signal

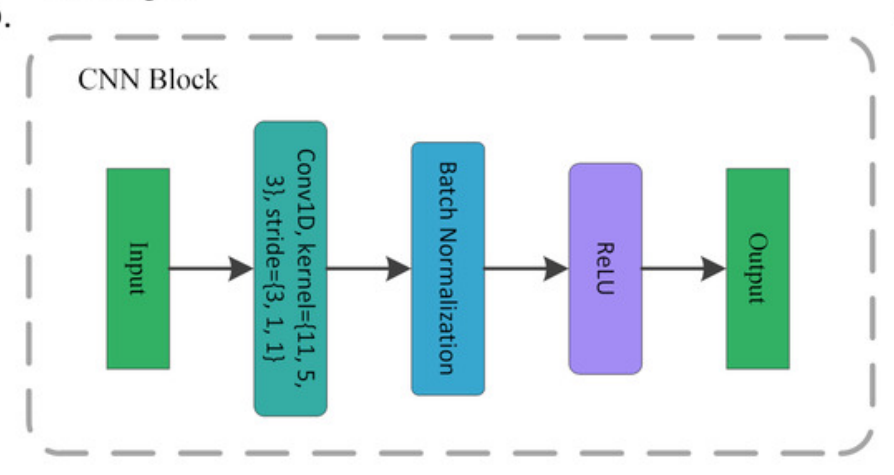

c.

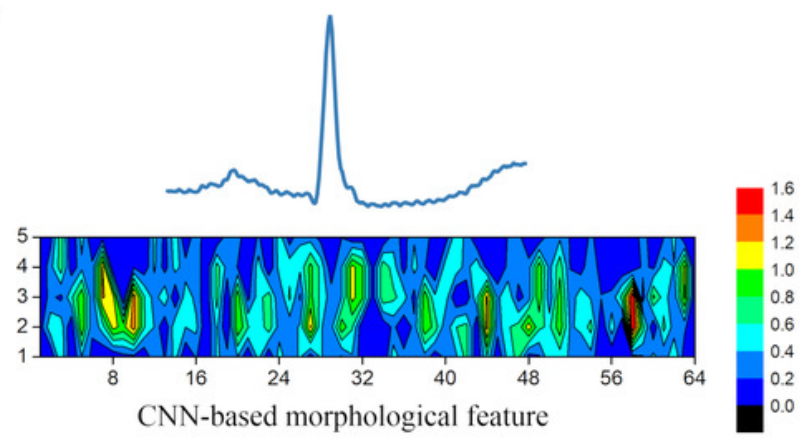


Figure 2

Example of baseline wandering removal.

a) Raw ECG signal. b) Raw heartbeat. c) Baseline-corrected ECG signal. d) Baseline-corrected heartbeat. It is easy to notice that after removing the baseline wandering, the heartbeat is shifted to zero.

a.

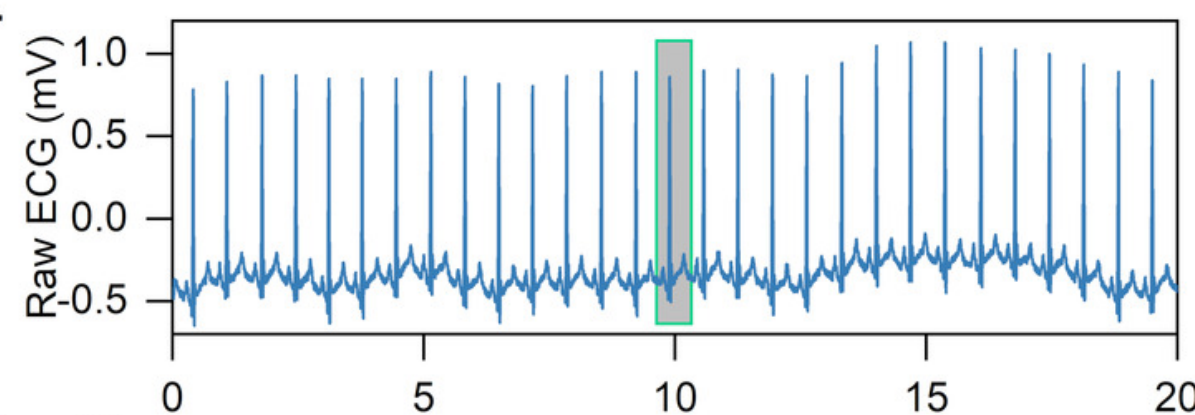

C.

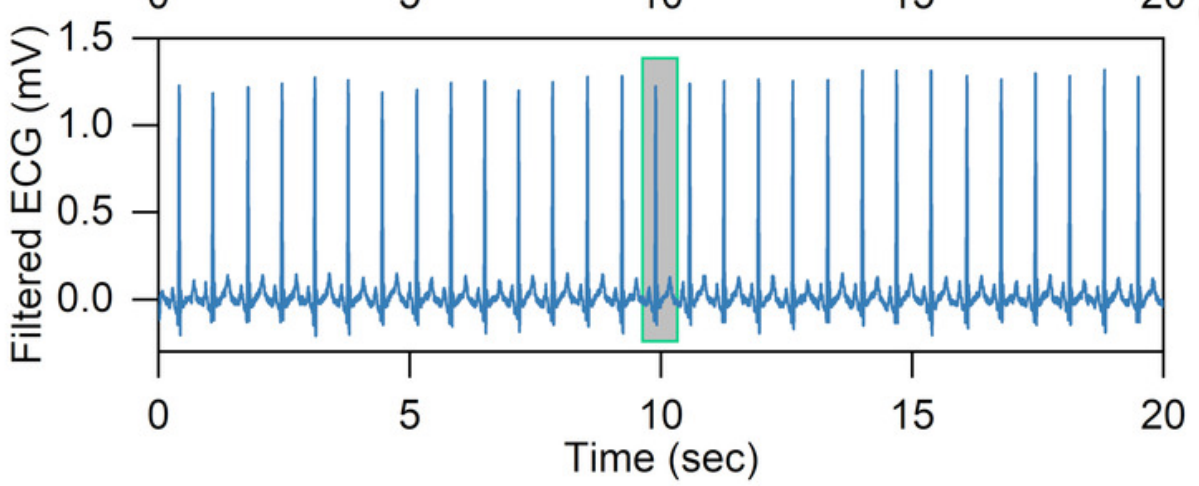

b.

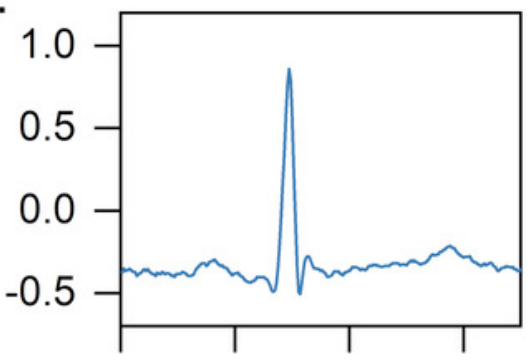

d.

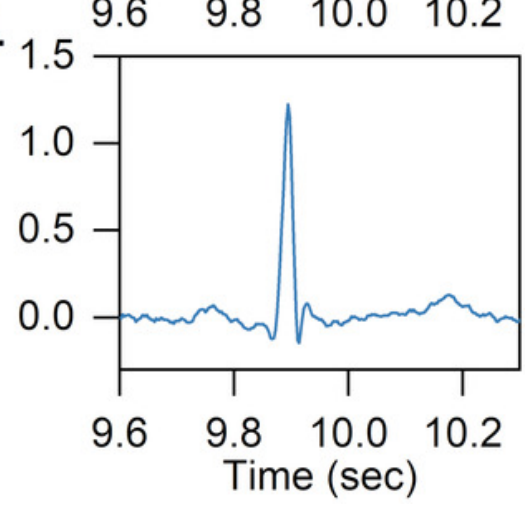


Figure 3

The relationship between the modulating factor $\gamma$ and the cost of the well-classified examples (Lin et al. 2017) .

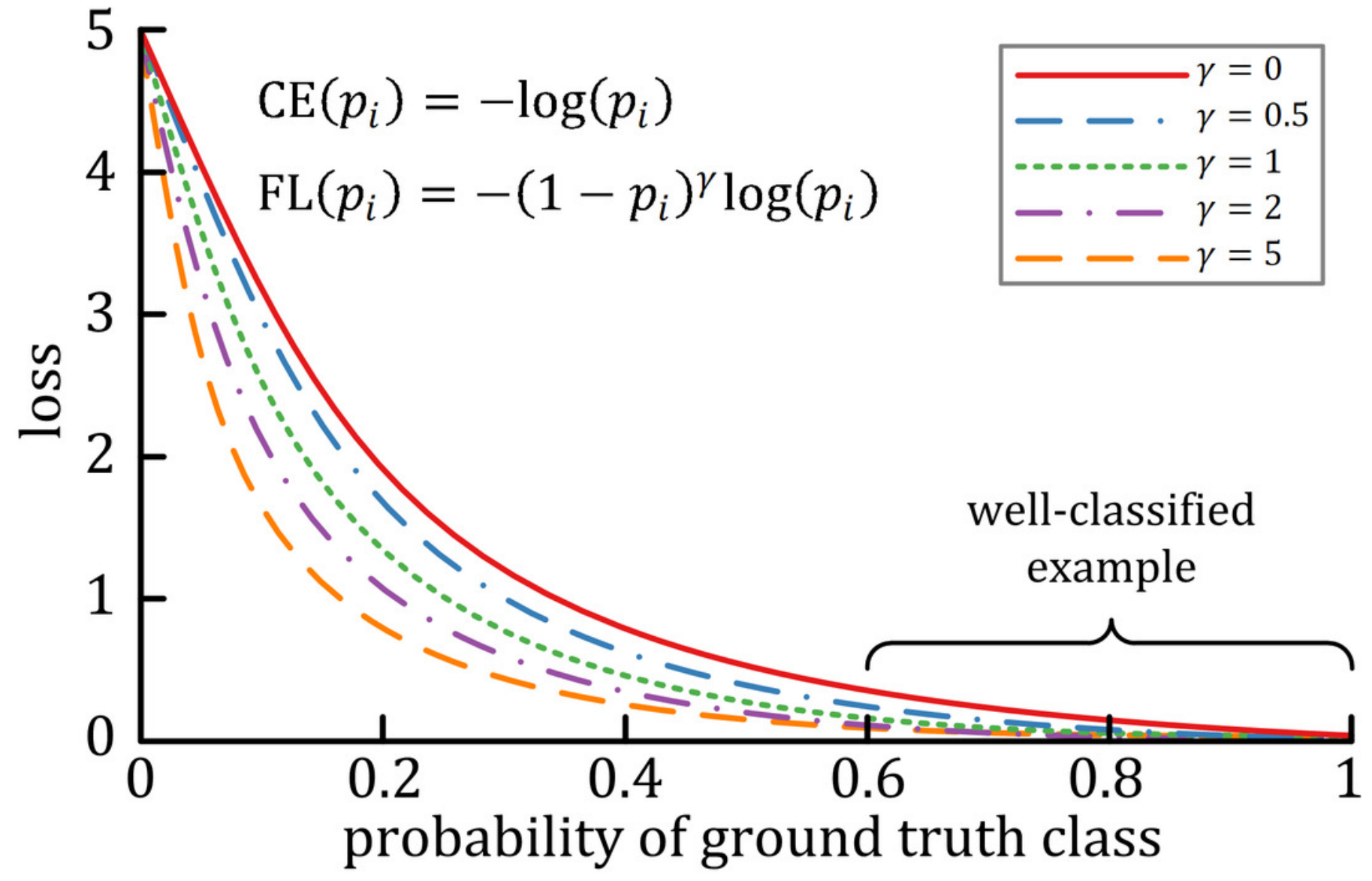




\section{Table $\mathbf{1}$ (on next page)}

The detailed parameters of our proposed deep neural network. 


\begin{tabular}{|c|c|c|c|c|c|c|c|}
\hline Layers & Layer Name & Kernel Size & $\begin{array}{l}\text { No. of } \\
\text { Filters }\end{array}$ & Stride & Output Shape & $\begin{array}{l}\text { No. of Trainable } \\
\text { Parameters }\end{array}$ & $\begin{array}{c}\text { No. of Non-trainable } \\
\text { Parameters }\end{array}$ \\
\hline 0 & Input $^{\mathrm{a}}$ & - & - & - & $200 \times 1$ & - & - \\
\hline 1 & 1D Convolution & 11 & 16 & 3 & $64 \times 16$ & 192 & - \\
\hline 2 & Batch Normalization & - & - & - & $64 \times 16$ & 32 & 32 \\
\hline 3 & ReLU & - & - & - & $64 \times 16$ & - & - \\
\hline 4 & Max-Pooling & 3 & - & 2 & $31 \times 16$ & - & - \\
\hline 5 & 1D Convolution & 5 & 32 & 1 & $27 \times 32$ & 2592 & - \\
\hline 6 & Batch Normalization & - & - & - & $27 \times 32$ & 64 & 64 \\
\hline 7 & ReLU & - & - & - & $27 \times 32$ & - & - \\
\hline 8 & Max-Pooling & 3 & - & 2 & $13 \times 32$ & - & - \\
\hline 9 & 1D Convolution & 3 & 64 & 1 & $11 \times 64$ & 6208 & - \\
\hline 10 & Batch Normalization & - & - & - & $11 \times 64$ & 128 & 128 \\
\hline 11 & ReLU & - & - & - & $11 \times 64$ & - & - \\
\hline 12 & Max-Pooling & 3 & - & 2 & $5 \times 64$ & - & - \\
\hline 13 & Flatten & - & - & - & 320 & - & - \\
\hline 14 & Input $2^{b}$ & - & - & - & 4 & - & - \\
\hline 15 & Concatenate & - & - & - & 324 & - & - \\
\hline 16 & Dense & - & - & - & 64 & 20800 & - \\
\hline 17 & Dense & - & - & - & 4 & 260 & - \\
\hline
\end{tabular}

${ }^{a}$ refers to the raw signal of the heartbeat. The morphological features of the heartbeat will be obtained through the CNN architecture.

$\mathrm{b}$ is the RR interval features of the heartbeat. It will be combined with the CNN-based morphological features to build the final classification model. 


\section{Table 2 (on next page)}

Mapping of AAMI classes and MIT-BIH arrhythmia heartbeat types. 


\begin{tabular}{|c|c|c|}
\hline AAMI Classes & MIT-BIH Types & $\begin{array}{l}\text { MIT-BIH } \\
\text { Annotate }\end{array}$ \\
\hline \multirow[t]{5}{*}{ Normal $(\mathrm{N})$} & Normal beat (NOR) & $\mathrm{N}$ \\
\hline & Nodal (junctional) escape beat (NE) & $\mathrm{j}$ \\
\hline & Atrial escape beat (AE) & e \\
\hline & Right bundle branch block beat (RBBB) & $\mathrm{R}$ \\
\hline & Left bundle branch block beat (LBBB) & $\mathrm{L}$ \\
\hline \multirow{4}{*}{$\begin{array}{l}\text { Supraventricular ectopic beat } \\
\text { (SVEB) }\end{array}$} & Aberrated arial premature beat (aAP) & a \\
\hline & $\begin{array}{l}\text { Premature or ectopic supraventricular beat } \\
\text { (SP) }\end{array}$ & $\mathrm{S}$ \\
\hline & Nodal (junctional) premature beat (NP) & $\mathrm{J}$ \\
\hline & Atrial premature beat (AP) & A \\
\hline \multirow[t]{2}{*}{ Ventricular ectopic beat (VEB) } & Ventricular escape beat (VE) & $\mathrm{E}$ \\
\hline & Premature ventricular contraction (PVC) & V \\
\hline Fusion beat (F) & Fusion of ventricular and normal beat (fVN) & $\mathrm{F}$ \\
\hline \multirow[t]{3}{*}{ Unknown beat $(\mathrm{Q})$} & Unclassifiable beat (U) & Q \\
\hline & Fusion of paced and normal beat (fPN) & $\mathrm{f}$ \\
\hline & Paced beat $(\mathrm{P})$ & / \\
\hline
\end{tabular}




\section{Table 3(on next page)}

Detailed breakdown of the dataset. 


\begin{tabular}{lccccc}
\hline \hline \multirow{2}{*}{ Dataset } & \multicolumn{4}{c}{ No. Of Samples Per AAMI Class } & \multirow{2}{*}{ Total } \\
\cline { 2 - 5 } & $\mathbf{N}$ & SVEB & VEB & F & \\
\hline DS1 & 45,824 & 943 & 3,788 & 414 & 50,969 \\
DS2 & 44,218 & 1,836 & 3,219 & 388 & 49,661 \\
Total (DS1+DS2) & 90,042 & 2,779 & 7,007 & 802 & 100,630 \\
\hline \hline
\end{tabular}

1 


\section{Table 4 (on next page)}

Performance comparison of our proposed method with existing works in SVEB and VEB classes. 


\begin{tabular}{|c|c|c|c|c|c|c|c|c|c|}
\hline \multirow{2}{*}{ Methods } & \multicolumn{4}{|c|}{ SVEB } & \multicolumn{3}{|l|}{$\square$} & \multicolumn{2}{|l|}{ VEB } \\
\hline & PPV (\%) & SE (\%) & F1 (\%) & Accuracy (\%) & $\bar{\square}$ & PPV (\%) & SE (\%) & F1 (\%) & Accuracy (\%) \\
\hline de Chazal et el. & 38.53 & 75.98 & 51.13 & 94.61 & & 81.67 & 80.31 & 80.98 & 97.62 \\
\hline Chen et al. & 38.40 & 29.50 & 33.36 & 95.34 & & 85.25 & 70.85 & 77.38 & 97.32 \\
\hline Zhang et al. & 35.98 & 79.06 & 49.46 & 93.33 & & 92.75 & 85.48 & 88.96 & 98.63 \\
\hline Mar et al. & 33.53 & 83.22 & 47.80 & 93.28 & & 75.89 & 86.75 & 80.96 & 97.35 \\
\hline Liu et al. & 39.87 & 33.12 & 36.18 & 95.49 & $\square$ & 76.51 & 90.20 & 82.79 & 97.45 \\
\hline Garcia et al. & 53.00 & 62.00 & 57.15 & - & $\square$ & 59.40 & 87.30 & 70.70 & - \\
\hline Our Proposed & 68.34 & 81.37 & 74.29 & 97.92 & $\square$ & 91.12 & 93.72 & 92.40 & 99.00 \\
\hline
\end{tabular}




\section{Table 5 (on next page)}

Performance comparison of our proposed method with existing works in all four classes. 


\begin{tabular}{|c|c|c|c|c|c|c|c|c|c|c|}
\hline \multirow{2}{*}{ Methods } & \multirow{2}{*}{ Accuracy $^{a}$} & \multirow{2}{*}{ Macro-F1 $1^{b}$} & \multicolumn{2}{|c|}{$\mathbf{N}$} & \multicolumn{2}{|c|}{ SVEB } & \multicolumn{2}{|c|}{ VEB } & \multicolumn{2}{|c|}{$\mathbf{F}$} \\
\hline & & & PPV (\%) & SE (\%) & $\square$ PPV (\%) & SE (\%) & $\square$ PPV (\%) & SE (\%) & $\square$ PPV (\%) & SE (\%) \\
\hline de Chazal et el. & 86.24 & 60.12 & 99.17 & 87.06 & 38.53 & 75.98 & 81.67 & 80.31 & 8.57 & 89.43 \\
\hline Chen et al. & 93.14 & 51.91 & 95.42 & 98.42 & 38.40 & 29.50 & 85.25 & 70.85 & 0.00 & 0.00 \\
\hline Zhang et al. & 88.34 & 64.02 & 98.98 & 88.94 & 35.98 & 79.06 & 92.75 & 85.48 & 13.73 & 93.81 \\
\hline Mar et al. & 88.99 & 62.24 & 99.12 & 89.64 & 33.53 & 83.22 & 75.89 & 86.75 & 16.57 & 61.08 \\
\hline Liu et al. & - & 58.50 & 96.66 & 94.06 & 39.87 & 33.12 & 76.51 & 90.20 & 12.99 & 40.72 \\
\hline Garcia et al. & 92.40 & 55.95 & 98.00 & 94.00 & 53.00 & 62.00 & 59.40 & 87.30 & - & - \\
\hline Our Proposed & 92.53 & 66.09 & 98.20 & 93.67 & 68.34 & 81.37 & 91.12 & 93.72 & 1.06 & 5.41 \\
\hline
\end{tabular}

a Accuray $=\left(\mathrm{TP}_{\mathrm{N}}+\mathrm{TP}_{\mathrm{SVEB}}+\mathrm{TP}_{\mathrm{VEB}}+\mathrm{TP}_{\mathrm{F}}\right) /$ number of testing heartbeats

${ }^{\mathrm{b}}$ refers to the average $\mathrm{fl}$-score of four AAMI classes. 


\section{Table 6(on next page)}

Performance comparison of focal loss and cross-entropy loss. 


\begin{tabular}{|c|c|c|c|c|c|}
\hline \multirow{2}{*}{ Methods } & \multirow{2}{*}{ AAMI Class } & \multicolumn{4}{|c|}{ Performance Metrics } \\
\hline & & PPV (\%) & SE (\%) & F1 (\%) & Accuracy (\%) \\
\hline \multirow{5}{*}{ Focal Loss } & $\mathrm{N}$ & 98.20 & 93.67 & 95.88 & 92.84 \\
\hline & SVEB & 68.34 & 81.37 & 74.29 & 97.92 \\
\hline & VEB & 91.12 & 93.72 & 92.40 & 99.00 \\
\hline & $\mathrm{F}$ & 1.06 & 5.41 & 1.77 & 95.31 \\
\hline & Average & 64.68 & 68.55 & 66.09 & 96.27 \\
\hline \multirow{5}{*}{ Cross-Entropy Loss } & $\mathrm{N}$ & 98.11 & 92.42 & 95.18 & 91.67 \\
\hline & SVEB & 57.11 & 80.72 & 66.89 & 97.05 \\
\hline & VEB & 83.95 & 93.45 & 88.44 & 98.42 \\
\hline & $\mathrm{F}$ & 0.11 & 0.52 & 0.18 & 95.54 \\
\hline & Average $^{a}$ & 59.82 & 66.77 & 62.67 & 95.67 \\
\hline
\end{tabular}

${ }^{a}$ refers to the average value of the corresponding metrics of four AAMI classes.

1 


\section{Table 7 (on next page)}

Confusion matrix of focal loss and cross-entropy loss. 


\begin{tabular}{|c|c|c|c|c|c|c|c|c|c|c|c|c|c|}
\hline \multirow{2}{*}{$\square$} & \multirow{2}{*}{ Focal Loss } & \multicolumn{4}{|c|}{ Predicted Class } & \multirow{2}{*}{ Total } & & \multirow{2}{*}{ Cross-Entropy Loss } & \multicolumn{4}{|c|}{ Predicted Class } & \multirow{2}{*}{ Total } \\
\hline & & $\mathbf{N}$ & SVEB & VEB & $\mathbf{F}$ & & & & $\mathbf{N}$ & SVEB & VEB & $\mathbf{F}$ & \\
\hline \multirow{4}{*}{ 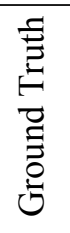 } & $\mathrm{N}$ & 41420 & 671 & 206 & 1921 & 44218 & & $\mathrm{~N}$ & 40866 & 1082 & 462 & 1808 & 44218 \\
\hline & SVEB & 282 & 1494 & 57 & 3 & 1836 & & SVEB & 246 & 1482 & 95 & 13 & 1836 \\
\hline & VEB & 141 & 21 & 3017 & 40 & 3219 & & VEB & 173 & 31 & 3008 & 7 & 3219 \\
\hline & $\mathrm{F}$ & 336 & 0 & 31 & 21 & 388 & & $\mathrm{~F}$ & 368 & 0 & 18 & 2 & 388 \\
\hline$\square$ & Total & 42179 & 2186 & 3311 & 1985 & 49661 & $\square$ & Total & 41653 & 2595 & 3583 & 1830 & 49661 \\
\hline
\end{tabular}

